\title{
配网检修安全技术措施探讨
}

\author{
叶凯朱F航
}

国网山东省电力公司商河县供电公司

DOI:10.32629/hwr.v3i3.1969

[摘 要] 随着我国经济的不断增长推动了电力技术的发展, 配电设备和线路的检修维护已经成为电网中最主要的工作, 通常 的配网在检修过程中会存在着各种各样的安全问题,需要通过科学合理的技术对其进行检修和管理,然后对其检修措施加以控 制,配网检修的安全管理也应该采取固定的操作规程和标准,这样才能够及时发现问题并选择合适的方式去检测。

[关键词] 配网; 检修; 安全技术; 措施

配网检修首先需要注意的就是绝缘防护措施, 要积极预 防相关的工作人员发生电击等危险的事故, 因此要选择一些 质量好, 而且防护全面的工具, 规范其在检修过程中的使用 方法, 并注意在过程中的维护和管理, 要充分认识到其中存 在的各种各样的安全隐患, 然后加强合理科学的规划和改造, 对其检修安全技术措施进行创新, 坚持技术先行的这种理念, 这样才是提高配网检修安全的重要措施。

\section{1 配电网检修安全工作的目的}

配电网检修安全工作最主要是为了保持和维护设备的 基本功能, 而采取的一项技术方面的工作, 他的任务, 通常包 括以下几个方面, 有检查, 维护, 修理和更新等等, 我国是世 界上带电作业项目, 作业人数以及出现作业事故最多的国家 之一, 配网检修事故直接会关系到整个绝缘环境的状况, 在 工作过程中, 由于导线的间距太近而引起的放电现象, 作业 过程中就会触碰到接近带电体的物质, 进而发生事故, 但事 故最根本的原因还是人员素质的问题, 包括相关的工作人员 没有按照操作规程进行操作, 管理人员未能对监督管理工作 进行全面的实行, 因此加强检修安全工作已经成为目前配电 网减少事故发生率最不可忽视的, 也是能够全面提高检修水 平的重要措施, 检修安全工作, 最主要的原则还是安全, 在保 障安全的基础之上, 然后进行预防对其进行综合的管理, 然 后根据检修对象和周围的环境的特点, 严格落实设备安全管 理责任制, 抓好配网检修过程中的安全技术的措施, 从而可 以不断的发现其中存在的隐患, 进而进行控制, 预防检修事 故的发生, 要充分认识到其中存在的各种各样的安全隐患, 然后加强合理科学的规划和改造, 对其检修安全技术措施进 行创新, 坚持技术先行的这种理念, 这样就可以更好的确保配 电网和检修人员的人身安全, 从而提高整个电网的供电质量。

\section{2 配电检修安全的措施探讨}

\section{1 绝缘防护用具的选择}

配网的绝缘防护措施是其检修过程中保障工作人员生 命安全最重要的措施之一, 因而在选取防护用具的过程中, 就需要多加谨慎, 这样才能够提高整体的安全性, 当人体和 带电体保持安全距离时, 且采用一些良好的绝缘防护用具, 就能够在很大程度上确保工作人员的人身安全, 绝缘防护用
具, 最主要是指操作过程中所涉及到的一些绝缘服装, 遮蔽 罩, 以及操作杆等等, 在选择上应该全面的了解工作的性质 以及电气与物理的性能, 依据安全生产规定进行严格的挑 选。绝缘防护用具的具体类型有很多, 包括一些绝缘安全器 具, 一般安全器具, 安全围栏和个人防护用品等等。这些绝缘 防护用具通常应该根据检修工作人员所, 需要使用的型号和 尺寸进行量身定做, 保持与厂家的长期合作, 然后根据国家 和行业以及企业的相关标准来制定最为安全的防护工具, 对 于一些进口的防护设备, 还应该考虑它是否符合目前我国的 标准, 也应该认真的进行接收验收和检查, 按照具体的规章 标准来进行操作。

\section{2 绝缘防护用具的使用}

选择一些合格的绝缘防护用具之后, 还应该对其具体的 使用方法进行仔细的探索, 进行科学规范的操作和使用, 因 此首先应该检查防护用具是否按照规定的标准, 以及相关的 电气试验和机械试验, 检查其是否存在一些损坏或磨损的状 况, 掌握其正确使用的方法。使用防护用具进行作业的过程, 可以探究出很多方法, 比如以绝缘工具为主, 绝缘用具为辅 的间接操作法, 或者是以绝缘壁, 或绝缘平台为主, 绝缘用具 为辅的直接作业法。这两种方式都可以在作业过程中交替使 用, 通常情况下间接作业法, 各种部分通过绝缘手套绝缘以 及绝缘靴等防护用具来确保电梯和接地体之间保持一定的 安全距离, 保证人们可以不用直接的触碰到发电体而发生事 故, 直接作业法, 主要是借助一些高空的作业车作为设备进 行检修和作业的工具, 作业人员戴绝缘手套和带电体之间就 会处于同等的地位。

\section{3 绝缘防护用具的维护}

绝缘防护用具的性能是直接关系到整个作业人员的人 身安全的, 其检修维护的保养, 也成为确保其性能的最主要 的工作, 维护对象主要分为个人维护用具以及大型的防护设 备的维护, 个人防护用具主要是存在一些表面的污染受潮破 损等情况, 这样就可能会发生一些电流的泄露, 导致电击事 故的出现, 目前个人防护用具的材质大多数是塑料或者橡胶, 这些材料, 极其容易被破坏, 因此作业人员应该加强使用前 和使用后的一些检查维修工作, 确保其安全性, 但是由于这 
类用具透气性也很差, 因此在工作人员, 水下作业的时候很 难保障其绝缘性, 因此需要及时的用干毛巾进行擦拭, 进行 安全用具的定期维护和保养, 大型的一些防护设备为例, 我国 大多数这类产品是引进外国的行业维护的标准, 存在很多不 规范的现象, 建议在维护的过程中应该定期检查维护的程序。

\section{4 检修设备防护措施}

配网检修应该对各个方面的关键设备的线路, 采取严格 的检修测试, 通常包括环网柜, 电缆分接箱, 美式箱变, 架空 绝缘线, 电缆线等关键设备, 环网柜是由一些高压的开关设 备控制的供电设备, 其核心部分是复合开关和熔断器所占有 的体积非常小, 而且价格十分经济, 能够改善供电性能, 在对 环网柜的防护测试中, 需要设置合格的接地设备, 改变以往 临时接地的做法, 同时也减小了停电的范围, 配备可靠的验 电设备, 根据国家电网的规定, 对无法直接验电的设备要进 行间接的用电检查刀闸机械指示, 电气指示等仪表的情况, 这样才能够判断验对象是否完好。

2.4 .1 电缆分接箱的维护措施

电缆分接箱的电缆线路中, 存在着很多分解的支路, 这 些绝缘设备可以实现电力电缆间的物理连接, 有着较高的安 全性, 其维护也十分简便, 绝缘性能良好, 价格经济。现在很 多的电缆分接箱可以分为带开关和不带开关的两类, 进线带 开关的分接箱有接地刀闸和带电显示器, 它的安全措施与环 网柜有很多类似的地方, 不带开关的分接箱配有带电显示器, 但是没有接地刀闸的配备, 电网检修时需要临时的进行断电 接地, 这样才能够确保作业过程中的安全性问题。

\section{4 .2 架空线的维护措施}

架空线是由杆塔将导线架设在高空中的电能传输和绝 缘线路, 架空绝缘线和电缆线路在检修的过程中应该在唁电 以后适当的位置进行接地装置的安装, 目前的安装规定, 对 接地环等安全装置没有明确的要求, 因此就需要根据工程的 实践情况安装在柱上开关和若干垂直线感变压器庄头等处。 随着我国城乡电网的不断改造和升级, 配电网在现代发展的 水平越来越高, 社会对其检修的模式和管理都提出了更多新 的要求。

\section{4 .3 美式箱变的维护措施}

美式箱变是从欧式箱变发展而来的, 这是一种集成程度 相当高的箱变产品最主要的特点是, 其内部有高压电气开关 和变压器, 优点是, 其过载的能力相当强, 而且整体的体积小, 经济性能好, 美式箱变的安全防护措施通常有以下几个方面, 电缆进线是电缆头上应安装带电指示装备, 这样可以辅助其 判断是否有电箱变进线, 要留有足够的空间接头, 以便在接 地的过程中便于使用。

配网检修中重点需要注意的就是绝缘防护措施和设备 防护措施, 要充分认识到其中存在的各种各样的安全隐患, 然后加强合理科学的规划和改造, 对其检修安全技术措施进 行创新, 坚持技术先行的这种理念, 以此来引导安全管理模 式更进一步, 使之能够提高到更高的水平, 在配网的实际检 修工作当中, 提高检修安全水平的主要因素还是人员问题, 通过技术培训和上岗以后的各种制度的规定, 提高他们对工 作的熟练度和规范程度, 从而就能够改进整个操作的方法, 提高安全技术水平，也能在此基础上减少更多的事故发生。

\section{3 结束语}

根据以上的综合论述, 我们需要在配网工作过程中, 要 充分认识到其中存在的各种各样的安全隐患, 然后加强合理 科学的规划和改造, 对其检修安全技术措施进行创新, 坚持 技术先行的这种理念, 严格的按照相关的规程和标准, 发现 问题并及时进行解决, 预防一些事故的发生, 这样才能够在 确保安全的条件下使其发展得更加安全和经济, 同时要把检 修安全和绝缘防护措施作为工作的重点, 可以整体的提高安 全管理水平, 进而能够不断丰富检修的理论参考。

[参考文献]

[1]永街,更烧序,解小军.同新能开电网安全运行水平的 工作方法[J].电信技术,2017,(22)40:513.

[2]李里,张金睡石,吴丹.电力工程施工技术与管理 [J].企业 学报,2018,(4):290.

[3]李新,刘近无,袁立.配网电力工程的技求问题分析与 解决[J].北京电力高等科学校学组,2017,(2):57-58. 